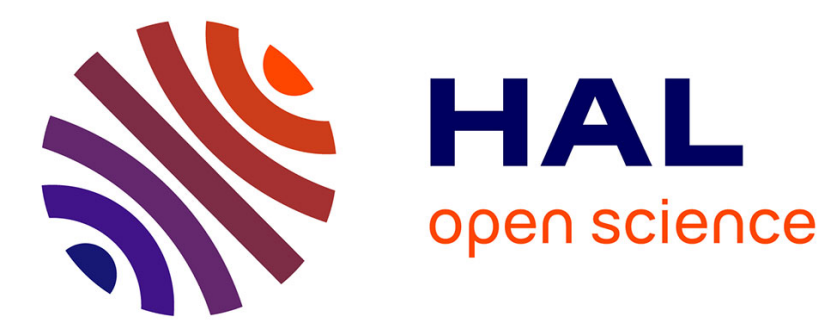

\title{
Quantitative and qualitative approach for shearwave elastography in superficial lymph nodes
}

Linda Chami, Alain Giron, Malek Ezziane, Veronique Leblond, Frédéric Charlotte, Claire Pellot-Barakat, Olivier Lucidarme

\section{To cite this version:}

Linda Chami, Alain Giron, Malek Ezziane, Veronique Leblond, Frédéric Charlotte, et al.. Quantitative and qualitative approach for shearwave elastography in superficial lymph nodes. 2021. hal-03115108

\author{
HAL Id: hal-03115108 \\ https://hal.science/hal-03115108
}

Preprint submitted on 19 Jan 2021

HAL is a multi-disciplinary open access archive for the deposit and dissemination of scientific research documents, whether they are published or not. The documents may come from teaching and research institutions in France or abroad, or from public or private research centers.
L'archive ouverte pluridisciplinaire HAL, est destinée au dépôt et à la diffusion de documents scientifiques de niveau recherche, publiés ou non, émanant des établissements d'enseignement et de recherche français ou étrangers, des laboratoires publics ou privés. 


\section{Quantitative and qualitative approach for shearwave elastography in superficial lymph nodes}

Linda Chami ${ }^{\mathrm{a}, \mathrm{b}}$, Alain Giron ${ }^{\mathrm{b}}$, Malek Ezziane ${ }^{\mathrm{a}}$, Véronique Leblond ${ }^{\mathrm{c}}$, Frédéric Charlotte ${ }^{\mathrm{d}}$, Claire Pellot-Barakat ${ }^{\mathrm{e}}$, Olivier Lucidarme ${ }^{\mathrm{a}, \mathrm{b}}$

\section{Affiliations:}

a Department of Radiology, Pitié-Salpêtrière Hospital, Sorbonne University, 47-83 Bd de l'Hôpital 75651 Paris cedex 13, France

${ }^{\mathrm{b}}$ LIB, INSERM, CNRS, UMR7371-U1146, Sorbonne University, 15 rue de l'Ecole de Médecine, 75006 PARIS, France

${ }^{c}$ Department of Haematology, Pitié-Salpêtrière Hospital, Sorbonne University, 47-83 Bd de l’Hôpital - 75651 Paris cedex 13, France

${ }^{\mathrm{d}}$ Department of Pathology, Pitié-Salpêtrière Hospital, Sorbonne University, 47-83 Bd de 1'Hôpital 75651 Paris cedex 13, France

${ }^{\text {e } B i o M a p s, ~ U n i v e r s i t y ~ P a r i s-S a c l a y, ~ C E A, ~ C N R S, ~ I N S E R M, ~ S e r v i c e ~ H o s p i t a l i e r ~ F r e ́ d e ́ r i c ~ J o l i o t, ~} 4$ place du général Leclerc, 91401 ORSAY France

\section{Corresponding Author:}

Claire Pellot-Barakat, $\mathrm{PhD}$

UMR BioMaps

University Paris-Saclay, CEA, CNRS, INSERM

4 place du Général Leclerc

91400 Orsay

France

Email : claire.barakat@inserm.fr

Tél : +330169867739 


\begin{abstract}
The diagnostic contribution of 2D-Shear Wave Elastography (SWE) in superficial lymph nodes (LN) management of any origin was evaluated on 222 patients referred for needle core biopsy. Conventional B-mode/Doppler ultrasound examinations (Conv-US) and SWE were performed for each patient. Quantitative SWE parameters and qualitative SWE map features were extracted. Carcinomas were found significantly stiffer than benign LN $(29.5 \pm 32.3 \mathrm{kPa}$ versus $6.7 \pm 12.3 \mathrm{kPa})$. Lymphomas exhibited intermediate stiffness $(11.4 \pm 5.2 \mathrm{kPa})$. Qualitative $\mathrm{SWE}$ analysis provided color patterns specific to histopathology (stiff rim, nodular and undetermined patterns related to malignancy and blue pattern to benignity). Adding SWE to Conv-US improved the sensitivity of LN diagnosis (from $81.1 \%$ to $92.0 \%$ ) but decreased its specificity (from $73.2 \%$ to $67.6 \%$ ) due to the high prevalence of lymphomas over carcinomas. Inter-observer agreement of quantitative SWE was good (ICC of 0.82). LN location and histology type were shown to influence the reported diagnostic performance of SWE.
\end{abstract}

\title{
Keywords
}

Diagnostic Ultrasound; Shear Wave Elastography; Lymph nodes; Lymphomas; Performance analysis

\section{Introduction}

Lymph nodes (LN) might be involved in several pathologies: infectious, malignant, hematological, immune, systemic and inflammatory diseases. Conventional ultrasound (Conv-US) imaging is commonly required as the first line modality for diagnostic work-up of superficial LN and US-guided biopsy remains the most cost-effective procedure for final diagnosis of superficial lesions (Balakrishnan et al., 2016; Han et al., 2018). Indication for biopsy relies classically on B-mode and color Doppler analysis however previous studies reported a sensitivity ranging from 78 to $96 \%$ and a specificity from 76 to 98\% (Ariji et al., 1998; Sato et al., 1998; Tschammler et al., 1996; Wu et al., 1998a, 1998b, 1998c) in differentiating benign from malignant nodes. This means that false negative 
and false positive results (between $2 \%$ and $24 \%$ ) are high enough to frequently indicate a biopsy, leading to a significant number of unnecessary biopsies. Hence, finding ways to improve the accuracy of conventional US would be helpful. Elastography could be one of these ways. Indeed, elastography techniques have emerged to help provide a non-invasive assessment of pathology based on tissue mechanical properties and improve lesion differentiation. Recent Shear Wave elastography (SWE) techniques provide an absolute elasticity assessment avoiding shortcomings of strain elastography and theoretically overcoming operator dependency (Bercoff et al., 2004). Most published studies have been focusing on SWE elasticity indices extracted in user-selected regions of interest as well as on specific clinical conditions (specific diseases in specific LN localizations; i.e : breast cancer and axillary LN, head and neck cancers LN, etc). They all show that malignant lymph nodes are significantly stiffer than benign LN (Bhatia et al., 2012; Chen et al., 2018; Choi et al., 2013; Desmots et al., 2016; Jung et al., 2015). However an overlap of stiffness values between the two groups exists, leading to various accuracies of SWE used alone. The color elasticity map has been very little exploited for LN diagnosis and only few recent studies showed that some SWE color patterns might be useful to discriminate LNs according to histology (Chae et al., 2019; Luo et al., 2019).

We hypothesize that the variability of clinical conditions (i.e, different pathologies, different LN localizations) might explain in part the discrepancy between reported diagnostic performances of SWE between studies.

The purpose of this study was first to evaluate how different malignant diseases might influence tissue stiffness estimates as well as SWE color elastograms in various locations and second to evaluate the added value of quantitative and qualitative SWE to conventional US in routine clinical practice.

\section{Material and methods}

This prospective study was approved by the institutional review board of our institution (ID RCB: 2015-A01315-44). From 2015 to 2017, 222 consecutive patients (113 men, 109 women), referred for 
needle core biopsy under ultrasound guidance for superficial lymph nodes were prospectively included. Oral informed consent was systematically requested whereas written informed consent was waived as SWE was part of the routine protocol for patients referred for US-guided biopsy. Patients with clinical and ultrasound characteristics suggestive of infectious disease such as abscess or tuberculosis were excluded.

All US examinations and US guided needle core biopsies were performed by the same senior radiologist with 15 years of expertise in US imaging in oncology. Biopsies were all performed with a 16- or 18-Gauge Adjustable Coaxial Temno® needle (Carefusion, San Diego, CA, USA). Histopathological results, available for all patients were considered the reference standard.

\section{Conventional Ultrasound}

The examinations were performed using an Aixplorer® machine (SuperSonic Imagine Ltd, Aix-enProvence, France) with a SL10-2 probe (SuperSonic Imagine Ltd, Aix-en-Provence, France) with a central frequency of $10 \mathrm{MHz}$. The following B-mode/color Doppler criteria suspicious of malignancy were noted: 1) loss of hilum, 2) markedly hypoechoic LN compared to neighboring muscles, 3) hyper vascularization (central, mixed or peripheral), 4) cystic changes or calcifications. LNs were considered malignant according to Conventional Ultrasound if at least two of those US suspicious criteria were present. The long axis/short axis diameter size ratio was also calculated.

\section{$2 D S W E$}

For SWE acquisitions, a standardized methodology was performed: the same SL10-2 probe (SuperSonic Imagine Ltd, Aix-en-Provence, France) was used for all LN and was placed along the long axis of the LN. The SWETM box was adjusted to include the LN plus some surrounding tissue. To ensure the quality of the SWE map, the acquisition was frozen after the stabilization of the 2D elasticity map display (about 10s), and the elasticity map with the best color filling and temporal stability was selected through the cineloop for further quantitative and qualitative analysis. 


\section{Quantitative analysis}

A round and as large as possible region of interest (ROI) including the LN was manually delineated according to the B-mode image while avoiding artifacts. In case of LNs with large and predominant fatty hilum, the cortex part was privileged as it is the most informative site. The rationale for choosing a large ROI including most of the LN served two purposes: i) derive quantitative parameters representative of the whole LN stiffness and heterogeneity, ii) limit the operator dependency, iii) limit biais. The quantification box provided the following Qbox values expressed in kilopascals ( $\mathrm{kPa}$ ): average value of stiffness (Emean), maximum value of stiffness (Emax), standard deviation (SD).

Three consecutive measurements were performed, each time repositioning the probe using the standardized procedure described above, and the average values of each Emean, Emax, and SD were then calculated from the three repetitions. After manually repositioning the probe, the SWETM box and ROI locations differed from one acquisition to another. The size of the SWETM box as well as the other physical parameters of the B-mode image did not change (focus, depth, gain, etc.).

Furthermore, a reproducibility study was conducted in a subgroup of 38 patients in whom three additional 2D SWE maps were obtained by a second radiologist blinded to the previous 2D SWE acquisitions. This second radiologist had less than one year of experience in SWE imaging. Exams were selected randomly for the reproducibility test. The histological distribution of this subset of 38 exams was 16 benign LNs, 17 lymphomas and 5 carcinomas. The subset had slightly more benign LNs (42.1\% vs $32 \%)$ and less carcinomas (13.2\% vs $20.3 \%)$ than the full set.

\section{Qualitative analysis}

The same colorbar scale ranging from 0 (dark blue) to $100 \mathrm{kPa}$ (dark red) was chosen for all the examinations 
The presence or the absence of the following color patterns was prospectively accessed by the operator at the time of acquisition:

- Homogeneous blue pattern: the whole elasticity map (LN and surrounding area) is homogeneously soft, colored in blue (Fig 1A-B)

- Nodular pattern: the lesion can visually be differentiated from the background (i.e. the target is softer or stiffer than the surrounding tissues or displayed void areas (uncolored)) (Fig 1C-D)

- Rim pattern: the LN might exhibit void areas or colors different from the neighboring tissue and a yellow to red rim of harder stiffness surrounding the $\mathrm{LN}$ is visible (Fig 1E-F).

- Undetermined pattern: the whole QBox is crossed by soft and stiff disorganized areas and possibly unfilled regions and none of the previously described patterns are visible (Fig 1G).

\section{Diagnostic strategy}

Sensitivities, specificities and accuracies of either separated or combined conventional US and SWE findings were assessed using different diagnostic work up strategies:

1. Conventional US alone (Conv-US) : LNs considered malignant if at least two suspicious criteria are present on conventional US (Sc) whatever the SWE findings.

2. Quantitative SWE alone (Quant SWE) : LNs considered malignant if stiffness estimates greater than an optimal threshold $\mathrm{E}_{\text {th }}$ (cut-off value determined using logistic regression).

3. Qualitative SWE alone (Qual SWE) : LNs considered malignant if rim and/or nodular pattern (nod) or undetermined pattern (undt) and benign if blue pattern.

4. Combination of conventional US with qualitative SWE: LNs found benign on convention US were reclassified as malignant if rim or nodular pattern or undetermined pattern were present

5. Combination of conventional US with quantitative SWE: LNs found benign on conventional US were reclassified as malignant when the stiffness estimates were greater than the threshold. 
6. For the combined strategies, benign LNs on conventional US were reclassified as malignant if the stiffness estimates were greater than the threshold and, if not, when the rim or nodular pattern or undetermined pattern were present.

Table 1 summarizes the different diagnostic strategies.

\section{Statistical Analysis}

All statistical analyses were performed using the JMP software (SAS Institute Inc., Cary, NC, USA). The statistical level of significance was set to $p=0.05$. Scores were reported as means and standard deviation and Pearson coefficient was used to evaluate correlations.

Comparison of quantitative parameters (SWE quantitative parameters, age ...) as function of the 3 histological groups (benign, lymphomas, carcinomas) were performed with a one-way Anova followed by post-hoc t-tests to depict origin of differences.

For comparison of categorical data (such as LN localization or SWE pattern) as function of histology, chi-square tests $(4 * 3)$ were used to see whether categories are distributed homogeneously among histology groups or whether a category is more frequent in one histology than the other.

In order to elaborate strategies for diagnosis, logistic regression using the histology classification as gold-standard (supervised learning) were performed to optimize the thresholds of each SWE quantitative parameters (ROC analysis). Similarly, logistic models incorporating quantitative, qualitative and conventional US information were processed in order to improve sensitivity/specificity balance.

The inter-observer agreement of quantitative and qualitative SWE were assessed using intraclass correlation and Cohen's kappa Statistic. The percentage agreement (PA or accuracy: number of identically-classified by raters / total number) between the observers was calculated. The qualitative SWE inter-observer reliability was measured using the Cohen's kappa Statistic K with 4 classes 
corresponding to the 4 patterns (blue, nodule, rim and undetermined) as well as with two classes, corresponding to benign diagnosis (blue pattern) and malignant diagnosis (the three other patterns) as described in the diagnostic strategy.

For the quantitative SWE inter-observer agreement, intra-class correlation was computed according to:

$$
I C C=\frac{\hat{\sigma}_{p}^{2}}{\hat{\sigma}_{p}^{2}+\hat{\sigma}_{o}^{2}+\hat{\sigma}_{p e}^{2}}
$$

with $\hat{\sigma}_{p}^{2}$ the variance estimated for patients, $\hat{\sigma}_{b}^{2}$ the variance estimated for the two observers) and $\hat{\sigma}_{p e}^{2}$ the variance estimated for pure error (based on the intra-observer variability between the 3 measurements)." It is based on a mixed model with patients as random factor and rater as a fixed factor. Intra-observer reliability of quantitative SWE was assessed by computing the Coefficient of Variation (CV : standard deviation / mean) between the three measurements performed by a same operator on a patient. It was computed using the following equation:

$$
C V=\frac{1}{N} \sum_{i=1}^{N} \frac{\sigma_{i}}{\mu_{i}}
$$

with $N$ the number of patients, $\sigma_{i}$ the standard deviation between the 3 measurements of patient $i$ and $\mu$ the mean of the three patient's measurements.

\section{Results}

\section{Population}

71 benign and 151 malignant LNs (106 LNs involved by lymphomas and 45 by metastatic carcinomas) were studied. Table 2 summarizes the characteristics of the population and the LNs studied as a function of the histological results. The age of the patients and the localization of LNs differed significantly according to histology. Malignant LNs were significantly more frequent in older patients and in the head and neck region than benign LNs $(\mathrm{p}<0.005)$. 
LNs located in the axilla area were deeper than other LNs with a distance from skin to LNs of $15 \mathrm{~mm}$ [13.3-16.8] 95\% CI for the axilla area, $11.3 \mathrm{~mm}$ [10-12.6] 95\% CI for the head and neck and $13.4 \mathrm{~mm}$ [11.5-15.3] 95\% CI for the inguinal area $(\mathrm{p}=0.01)$.

Primary cancers of the carcinoma group originated from breast (24\%), lung (13\%), thyroid (13\%), prostate $(7 \%)$, colon $(4 \%)$, stomach (4\%), kidney (4\%), and various other types $(24 \%)$.

\section{Conventional US}

Frequencies of Conventional US findings as well as long axis/short axis size ratios are displayed in Table 3. Benign LNs had significant higher long axis/short axis ratio than malignant LNs although in most cases malignant LNs also exhibited an oval shape (long axis/short axis ratio >1). Presence of malignant criteria and long axis/short axis ratio did not differ significantly between lymphomas and carcinomas. Accuracies, sensitivities and specificities of the presence of at least two malignant criteria for malignancy, lymphoma or carcinoma are summarized in Table 4.

\section{SWE Quantitative analysis}

Average values of all three quantitative 2D SWE parameters (Emean, Emax and SD) obtained as a function of histology are summarized in Table 3. All three quantitative parameters (Emean, Emax or SD values) provided the same diagnostic performances and were highly correlated with correlation coefficients: Emean/Emax: 0.9, Emean/SD: 0.79, Emax/SD: 0.95.

Averaged values of Emean were $11.4 \pm 5.2 \mathrm{kPa}, 16.7 \pm 12.3 \mathrm{kPa}$ and $29.5 \pm 32.3 \mathrm{kPa}$ for benign LNs, lymphomas and carcinomas respectively. The distribution of the parameter Emean as function of histology is illustrated on Fig 2.

Accuracies, sensitivities and specificities of the SWE parameters for malignancy, lymphomas or carcinomas are summarized in Table 4. 
ROC analysis of mean elasticity values (Emean) revealed an optimal threshold around $15.2 \mathrm{kPa}(\mathrm{CI}$ : 11.1, 22.3) (Sensitivity: 43.7\%, Specificity: 83.1\%, AUC: 0.66 (CI: 0.59, 0.73), PPV: 0.85, NPV: 0.42) for differentiating benign and malignant LNs (Fig 3). Although SWE values of lymphomas were lower than those of carcinomas, cut-off thresholds for differentiating benign vs. lymphoma and benign vs. carcinomas were also $15.2 \mathrm{kPa}$ (Sensitivity: $37.7 \%$, Specificity: $83.1 \%$, AUC: 0.63, PPV: 0.78, NPV: 0.48 for differentiating benign LNs and lymphomas and Sensitivity: 57.8\%, Specificity: $83.1 \%$, AUC: 0.72, PPV: 0.68, NPV: 0.76 for differentiating benign LNs and carcinomas).

The overall inter-observer agreements of the average values of quantitative 2D SWE parameters obtained on a subset of data by two independent observers were $0.82,0.79$ and 0.82 (ICC agreement) for Emean, Emax and SD respectively. For the intra-observer reproducibility, the CV of Emean, Emax and SD were $15 \%, 15 \%$ and $19 \%$ for the first operator (senior radiologist) and $23 \%, 23 \%$ and $33 \%$ for the second operator (junior radiologist).

As shown on Table 5, the location of the LNs influenced the diagnostic performances of quantitative SWE: lymphomas showed higher stiffness values in the neck than in other areas and mean stiffness values failed to distinguish LNs according to histology in the axilla area.

\section{SWE Qualitative analysis}

Each pre-determined SWE color pattern was significantly related to the histology type (Table 3). Nodular, Rim and undetermined patterns were related to malignancy and blue pattern has been found to be related to benignity. However, the blue pattern occurrence in lymphomas was also very high as shown in Fig. 4 as well as in Table 4 with 55 out of 106 lymphomas exhibiting a blue pattern.

Performances of each SWE color pattern for each histology type are summarized in Table 4. Nodular, rim and undetermined patterns were highly specific of malignancy but were neither accurate nor 
sensitive. On the other side, the homogeneous blue pattern indicated benignity with accuracy, sensitivity and specificity of $65.8 \%$ (146/222), 57.0\% (86/151) and 84.5\% (60/71), respectively.

In a randomly chosen sub-population of 145 LNs analyzed by a second radiologist, the number (proportion) of blue, nodule, rim and undetermined patterns was $46(32 \%), 49(34 \%), 25(17 \%)$ and $25(17 \%)$ respectively for the first observer and $36(25 \%), 52(36 \%), 32(22 \%), 25(17 \%)$ respectively for the second observer. The kappa for the observation of the four patterns was 0.48 (CI: $0.37 ; 0.59)$ and the percentage agreement was $62.1 \%$. In terms of diagnostic agreement with two classes (benign/malignant according to the qualitative SWE diagnostic strategy), the kappa was 0.76 (CI: 0.65; 0.88 ) and the percentage agreement $90 \%$.

\section{Diagnostic work-up}

Accuracies as well as sensitivities and specificities for malignancy of different diagnostic work-up strategies are summarized in Table 6. The combination of conv-US and qualitative SWE, conv-US and quantitative SWE and conv-US and both qualitative and quantitative SWE showed accuracies of $84.2 \%, 83.8 \%$ and $83.3 \%$ respectively.

\section{Discussion}

Reported diagnostic performance of SWE in lymph nodes have mainly focused on quantitative SWE, head and neck or axillary localizations and on metastatic diseases. Despite including various pathologies, we found, as already published by several groups, that malignant LNs were significantly stiffer than benign ones and, additionally, we found that lymphomas showed stiffness values intermediate between benign LNs and carcinomas. Our results are similar to those obtained by Zhang et al (Zhang et al., 2017) using a point SWE system and Chae using Toshiba SWE (Chae et al., 2019). Combination of quantitative SWE with conventional US increased the sensitivity to the detriment of specificity. However, contrarily to previous reports, the accuracy for diagnosing malignancy did not improve (Chae et al., 2019; Chang et al., 2018; Desmots et al., 2016; Seo and Sohn, 2018). 
Our results are consistent with some recent studies that found that quantitative SWE did not add predictive power to prediction models in the assessment of cervical lymph nodes (Heřman et al., 2019; Lo et al., 2019). The analysis of these results requires taking into consideration technical as well as clinical parameters. In our patients, the receiver operating curve identified an optimal threshold of Emean for malignancy of $15.2 \mathrm{kPa}$. This value is much lower than the ones published in the literature (between 18.4 and $31 \mathrm{kPa}$ ) (Bhatia et al., 2012; Chen et al., 2018; Choi et al., 2013; Desmots et al., 2016; Jung et al., 2015; Kim et al., 2018). Two explanations for this lower threshold can be brought forward: 1) Lymphomas constituted the majority of LNs in our study. The most predominant subtype of lymphomas were Non-Hodgkin lymphomas such as follicular and B cell lymphomas. These pathological LNs have a proliferation characterized by less fibrotic changes (desmoplastic reaction) than carcinomas. Consequently merging both lymphomas and carcinomas in the malignancy group led to decrease the threshold value compared to a population of exclusively metastatic LNs as usually considered in the literature (Bhatia et al., 2013, 2012; Chen et al., 2018; Choi et al., 2013; Desmots et al., 2016; Jung et al., 2015; Kim et al., 2018; Seo and Sohn, 2018; Suh et al., 2017; Ying et al., 2014). 2) To standardize our findings due to variability of the localization of the LNs, we used a lower frequency probe (10-2 MHz) than that used in most studies which lead to lower stiffness values (Chami et al., 2016).

Another factor to take into consideration is the stiffness measurement technique itself (i.e the size and the placement of the ROI) that might differ from one study to another. Indeed, the size of the ROI has been shown to be related to the diagnostic performance and reproducibility of SWE measurements (Lee et al., 2013; Moon et al., 2017; Seliger et al., 2017; Skerl et al., 2015). In our study, we preferred a ROI as large as possible due to the heterogeneity of the LNs and in order to include most of the LNs. The main advantage of this method is that it standardizes our methodology and limits operator dependency as the placement and the size of the ROI is adapted to the boundary of the target and not to a visual interpretation of the SWE color map. We also systematically performed 3 measurements 
that are averaged in order to minimize spatial and temporal variability. Indeed, our inter-observer reproducibility for stiffness measurements calculated on a subset of patient was good. The diagnostic value of each stiffness parameter remains under debate (Bhatia et al., 2012; Chen et al., 2018; Choi et al., 2013; Kim et al., 2018; Lee et al., 2013). However, using our standardized methodology for the ROI size and placement, we found that the three parameters (Emean, Emax and SD values) provided the same diagnostic performance.

Although our study included a relatively small number of cases of LNs of specific histological types and locations, we demonstrated that while the LNs morphology on gray scale US did not differ as function of the location, the localization of LNs might influence the diagnostic performance of SWE. The reason why lymphomas showed higher stiffness values in the neck rather than in other areas remains unclear. In addition, quantitative SWE failed to diagnose malignancy for the LNs localized in the axilla area $(\mathrm{p}>0.05)$. This result differs from studies which showed a benefit of SWE in the diagnosis of metastatic axillary LNs in breast cancer (Luo et al., 2019) and might be explained in part by the deeper location of LNs in the axilla area compared to other locations with a SWE signal loss and, in another part, by the low rate of metastatic disease $(n=9)$ in this group of patients.

These findings illustrate how the probe frequency, the location of the disease, the technique of SWE measurements and the histology type might influence the reported diagnostic performance of SWE. Our results show that in clinical practice, quantitative SWE combined with conventional US allowed to detect more pathological LNs to the detriment of specificity. Operators should also keep in mind that some lymphomas which might appear clearly pathological on gray scale US conversely might provide intermediate stiffness values using quantitative SWE.

A way to improve LN diagnostic would be to consider not only stiffness values, but also to try to take advantage of the stiffness mapping. Qualitative SWE has been little studied in LNs. In previous preliminary results, we reported for the first time how qualitative SWE might be related to histology in lymph nodes (Chami et al., 2015). More recently, some published studies proposed SWE color map 
patterns as biomarkers to differentiate LNs (Chae et al., 2019; Luo et al., 2019). In our study, all the strategies that combined quantitative and/or qualitative SWE to conventional ultrasound exhibited close performances: they improved the sensitivity with a loss of specificity and thus accuracy performance close to that of conventional US alone. The highest accuracy was obtained when combining qualitative SWE to Conventional US $(84.2 \%$ versus $83.8 \%$ and $83.3 \%$ for the other combinations) although this improvement is very mild. Interestingly this result agrees with the findings of a recent study by Luo et al showing that qualitative SWE classification combined with conventional US exhibited better diagnostic performance than quantitative SWE and conventional US for differentiating metastatic from benign reactive axillary lymph nodes in breast cancer (Luo et al., 2019). Color patterns can be identified on the elasticity map. Using the same color scale range of $0-100 \mathrm{kPa}$ to standardize our qualitative acquisitions, we described four SWE patterns that were found to be suggestive of malignancy or benignity with high specificities but low sensitivities.

Our "nodular pattern" that was found to be related to malignancy could be similar to the "markedly heterogeneous with some unfilled regions" pattern described by Bhatia et al (Bhatia et al., 2016), the "heterogeneous pattern" described by Luo for metastatic disease in breast cancer (Luo et al., 2019), or the "large red area" and "almost red" patterns described by Chae (Chae et al., 2019). The "rim pattern" that was also suggestive of malignancy has been reported in primary breast cancer. It was interpreted as a marker of a desmoplastic reaction or the infiltration of cancer cells into the interstitial surrounding tissues (Chen et al., 2016; Cong et al., 2017a; Lee et al., 2017; Park et al., 2018; Zhou et al., 2014). Recently Luo et al. (Luo et al., 2019) described "a colored area at the margin" in LNs which is similar to our "rim pattern". Finally, the homogeneous blue pattern has been found to be related to benignity similarly to previous reports (Cong et al., 2017b; Luo et al., 2019; Park et al., 2018) but this sign showed a limited interest to distinguish benign LNs and lymphomas.

The main criticism that could be addressed to the qualitative analysis is that it is inherently subjective and even more since color patterns might be temporally instable. Indeed, some unfilled areas inside 
the elasticity map can also appear and disappear. Their signification remains unclear as they represent regions where shear wave velocity could not be measured, possibly due to the presence of necrosis or technical limitations. A moderate inter-observer agreement in the description of the four patterns, according to Cohen's interpretation of kappa values, was found between observers $(\mathrm{K}=0.48)$. However, the diagnostic ability of the technique was not penalized as the evaluation of the interobserver diagnostic reproducibility (when considering two color pattern groups corresponding to benign/malignant), showed a substantial agreement $(K=0.65)$.

SWE performances for LNs diagnosis are usually reported for a specific location and disease. In the current study, the same protocol and methods were used for mixed locations and various histologies allowing to highlight that, in clinical practice, the SWE findings should be interpretated depending on clinical conditions (location of the LNs, ratio of lymphomas versus carcinomas) and that a standardized methodology is needed. Our study confirms that SWE color map exhibits some color patterns which might be an interesting imaging biomarker for LN diagnosis. Indeed, combined qualitative SWE and conventional US provided the highest effectiveness for LN diagnosis. Operators should also keep in mind that when the expected prevalence of lymphomas is high, the addition of SWE to conventional US might have a limited interest. Qualitative SWE should be further investigated either explicitly as in the current study, through texture analysis and radiomics approaches or through unsupervised approaches such as deep learning.

\section{Acknowledgments}

Research was partly funded by the French program "Investissement d'Avenir" run by the 'Agence Nationale pour la Recherche' (grant 'Infrastructure d'avenir en Biologie Santé -ANR-11-INBS0006'). 


\section{References}

Ariji, Y., Kimura, Y., Hayashi, N., Onitsuka, T., Yonetsu, K., Hayashi, K., Ariji, E., Kobayashi, T., Nakamura, T. Power Doppler sonography of cervical lymph nodes in patients with head and neck cancer. AJNR. American journal of neuroradiology 1998:19:303-7.

Balakrishnan, M., Souza, F., Muñoz, C., Augustin, S., Loo, N., Deng, Y., Ciarleglio, M., GarciaTsao, G. Liver and Spleen Stiffness Measurements by Point Shear Wave Elastography via Acoustic Radiation Force Impulse: Intraobserver and Interobserver Variability and Predictors of Variability in a US Population. J Ultrasound Med 2016;35:2373-2380.

Bercoff, J., Tanter, M., Fink, M.. Supersonic shear imaging: a new technique for soft tissue elasticity mapping. IEEE transactions on ultrasonics, ferroelectrics, and frequency control 2004;51:396-409.

Bhatia, K.S.S., Cho, C.C.M., Tong, C.S.L., Yuen, E.H.Y., Ahuja, A.T. Shear wave elasticity imaging of cervical lymph nodes. Ultrasound in Medicine and Biology 2012;38:195-201.

Bhatia, K.S.S., Lam, A.C.L., Pang, S.W.A., Wang, D., Ahuja, A.T. Feasibility Study of Texture Analysis Using Ultrasound Shear Wave Elastography to Predict Malignancy in Thyroid Nodules. Ultrasound Med Biol 2016; 42(7):1671-80.

Bhatia, K.S.S., Lee, Y.Y.P., Yuen, E.H.Y., Ahuja, A.T. Ultrasound elastography in the head and neck. part II. accuracy for malignancy. Cancer Imaging 2013;13:260-276. https://doi.org/10.1102/1470-7330.2013.0027

Chae, S.Y., Jung, H.N., Ryoo, I., Suh, S., 2019. Differentiating cervical metastatic lymphadenopathy and lymphoma by shear wave elastography. Sci Rep 2019;9(1):12396. https://doi.org/10.1038/s41598-019-48705-0

Chami, L., Yue, J.L., Lucidarme, O., Lefort, M., Pellot-Barakat, C. Feasibility of liver shear wave elastography with different transducers, in: 2016 Ieee International Ultrasonics Symposium (Ius). Ieee, New York. 
Chami L., Pellot-Barakat C., Gabarre J., Giron A., Charlotte F., Leblond V., Lucidarme O. Usefulness of shearwave elastography in ultrasound imaging of superficial lymph nodes: Comparison with histological findings. Eur J Cancer 2015;51(S133):720.

Chang, W., Tang, L., Lu, C., Wu, M., Chen, M., 2018. Shear wave elastography in the evaluation of level VI lymph nodes in papillary thyroid carcinoma: combined with gray-scale ultrasound ex vivo. BMC Cancer 2018;18(1):1001.

Chen, B.-B., Li, J., Guan, Y., Xiao, W.-W., Zhao, C., Lu, T.-X., Han, F. The value of shear wave elastography in predicting for undiagnosed small cervical lymph node metastasis in nasopharyngeal carcinoma: A preliminary study. European Journal of Radiology 2018;103: $19-24$.

Chen, Y.-L., Chang, C., Zeng, W., Wang, F., Chen, J.-J., Qu, N. 3-Dimensional shear wave elastography of breast lesions: Added value of color patterns with emphasis on crater sign of coronal plane. Medicine 2016;95(39): e4877.

Choi, Y.J., Lee, J.H., Lim, H.K., Kim, S.Y., Han, M.W., Cho, K.J., Baek, J.H. Quantitative Shear Wave Elastography in the Evaluation of Metastatic Cervical Lymph Nodes. Ultrasound in Medicine and Biology 2013;39:935-940.

Cong, R., Li, J., Guo, S. A new qualitative pattern classification of shear wave elastograghy for solid breast mass evaluation. European journal of radiology 2017;87:111-119. https://doi.org/10.1016/j.ejrad.2016.12.021

Desmots, F., Fakhry, N., Mancini, J., Reyre, A., Vidal, V., Jacquier, A., Santini, L., Moulin, G., Varoquaux, A. Shear Wave Elastography in Head and Neck Lymph Node Assessment: Image Quality and Diagnostic Impact Compared with B-Mode and Doppler Ultrasonography. Ultrasound in Medicine and Biology 2016;42:387-398. 
Han, F., Xu, M., Xie, T., Wang, J.-W., Lin, Q.-G., Guo, Z.-X., Zheng, W., Han, J., Lin, X., Zou, R.H., Zhou, J.-H., Li, A.-H. Efficacy of ultrasound-guided core needle biopsy in cervical lymphadenopathy: A retrospective study of 6,695 cases. Eur Radiol 2018;28:1809-1817.

Heřman, J., Sedláčková, Z., Fürst, T., Vachutka, J., Salzman, R., Vomáčka, J., Heřman, M. The Role of Ultrasound and Shear-Wave Elastography in Evaluation of Cervical Lymph Nodes. Biomed Res Int 2019;4318251.

Jung, W.S., Kim, J.-A., Son, E.J., Youk, J.H., Park, C.S. Shear Wave Elastography in Evaluation of Cervical Lymph Node Metastasis of Papillary Thyroid Carcinoma: Elasticity Index as a Prognostic Implication. Annals of Surgical Oncology 2015;22:111-116.

Kim, H.J., Choi, I.H., Jin, S.-Y., Park, H.K., Byun, D.W., Suh, K., Yoo, M.H. Efficacy of ShearWave Elastography for Detecting Postoperative Cervical Lymph Node Metastasis in Papillary Thyroid Carcinoma. Int J Endocrinol 2018:9382649.

Lee, E.J., Jung, H.K., Ko, K.H., Lee, J.T., Yoon, J.H. Diagnostic performances of shear wave elastography: which parameter to use in differential diagnosis of solid breast masses? Eur Radiol 2013;23:1803-1811.

Lee, H.Y., Lee, J.H., Shin, J.H., Kim, S.Y., Shin, H.J., Park, J.S., Choi, Y.J., Baek, J.H. Shear wave elastography using ultrasound: effects of anisotropy and stretch stress on a tissue phantom and in vivo reactive lymph nodes in the neck. Ultrasonography (Seoul, Korea) 2017;36: 2532.

Lo, W.-C., Hsu, W.-L., Wang, C.-T., Cheng, P.-W., Liao, L.-J. Incorporation of shear wave elastography into a prediction model in the assessment of cervical lymph nodes. PLoS ONE 2019;14:e0221062.

Luo, S., Yao, G., Hong, Z., Zhang, S., Wang, W., Zhang, J., Zhang, Y., Wu, J., Zhang, L., Cheng, H., Hao, Y., Li, Y. Qualitative Classification of Shear Wave Elastography for Differential 
Diagnosis Between Benign and Metastatic Axillary Lymph Nodes in Breast Cancer. Front Oncol 2019:9:533.

Moon, J.H., Hwang, J.-Y., Park, J.S., Koh, S.H., Park, S.-Y. Impact of region of interest (ROI) size on the diagnostic performance of shear wave elastography in differentiating solid breast lesions. Acta Radiol 2017;59(6); 657-663.

Park, H.S., Shin, H.J., Shin, K.C., Cha, J.H., Chae, E.Y., Choi, W.J., Kim, H.H. Comparison of peritumoral stromal tissue stiffness obtained by shear wave elastography between benign and malignant breast lesions. Acta radiologica (Stockholm, Sweden : 1987) 2018;59(10):11681175.

Sato, N., Kawabe, R., Fujita, K., Omura, S. Differential diagnosis of cervical lymphadenopathy with intranodal color Doppler flow signals in patients with oral squamous cell carcinoma. Oral surgery, oral medicine, oral pathology, oral radiology, and endodontics 1998;86:482-488.

Seliger, G., Chaoui, K., Kunze, C., Dridi, Y., Jenderka, K.-V., Wienke, A., Tchirikov, M. Intra- and inter-observer variation and accuracy using different shear wave elastography methods to assess circumscribed objects - a phantom study. Med Ultrason 2017;19:357-365.

Seo, M., Sohn, Y.-M. Differentiation of benign and metastatic axillary lymph nodes in breast cancer: additive value of shear wave elastography to B-mode ultrasound. Clin Imaging 2018;50:258263.

Skerl, K., Vinnicombe, S., Giannotti, E., Thomson, K., Evans, A. Influence of region of interest size and ultrasound lesion size on the performance of 2D shear wave elastography (SWE) in solid breast masses. Clin Radiol 2015;70:1421-1427.

Suh, C.H., Choi, Y.J., Baek, J.H., Lee, J.H. The diagnostic performance of shear wave elastography for malignant cervical lymph nodes: A systematic review and meta-analysis. European Radiology 2017;27:222-230. 
Tschammler, A., Wirkner, H., Ott, G., Hahn, D. Vascular patterns in reactive and malignant lymphadenopathy. European radiology 1996;6:473-80.

Wu, C.H., Chang, Y.L., Hsu, W.C., Ko, J.Y., Sheen, T.S., Hsieh, F.J. Usefulness of Doppler spectral analysis and power Doppler sonography in the differentiation of cervical lymphadenopathies. AJR. American journal of roentgenology 1998a;171:503-9.

Wu, C.H., Hsu, M.M., Chang, Y.L., Hsieh, F.J. Vascular pathology of malignant cervical lymphadenopathy: qualitative and quantitative assessment with power Doppler ultrasound. Cancer 1998b;83:1189-96.

Wu, C.H., Shih, J.C., Chang, Y.L., Lee, S.Y., Hsieh, F.J. Two-dimensional and three-dimensional power Doppler sonographic classification of vascular patterns in cervical lymphadenopathies. Journal of ultrasound in medicine : official journal of the American Institute of Ultrasound in Medicine 1998c;17:459-64.

Ying, M., Bhatia, K.S.S., Lee, Y.P., Yuen, H.Y., Ahuja, A.T. Review of ultrasonography of malignant neck nodes: Greyscale, doppler, contrast enhancement and elastography. Cancer Imaging 2014;13:658-669.

Yoon, K., Jeong, W.K., Kim, Y., Kim, M.Y., Kim, T.Y., Sohn, J.H. 2-dimensional shear wave elastography: Interobserver agreement and factors related to interobserver discrepancy. PLoS ONE 12, 2017; e0175747.

Zhang, F., Zhao, X., Ji, X., Han, R., Li, P., Du, M. Diagnostic value of acoustic radiation force impulse imaging for assessing superficial lymph nodes A diagnostic accuracy study. Medicine 2017;96:1-7.

Zhou, J., Zhan, W., Chang, C., Zhang, X., Jia, Y., Dong, Y., Zhou, C., Sun, J., Grant, E.G. Breast lesions: evaluation with shear wave elastography, with special emphasis on the stiff rim sign. Radiology 2014;272:63-72. 


\section{Figures}
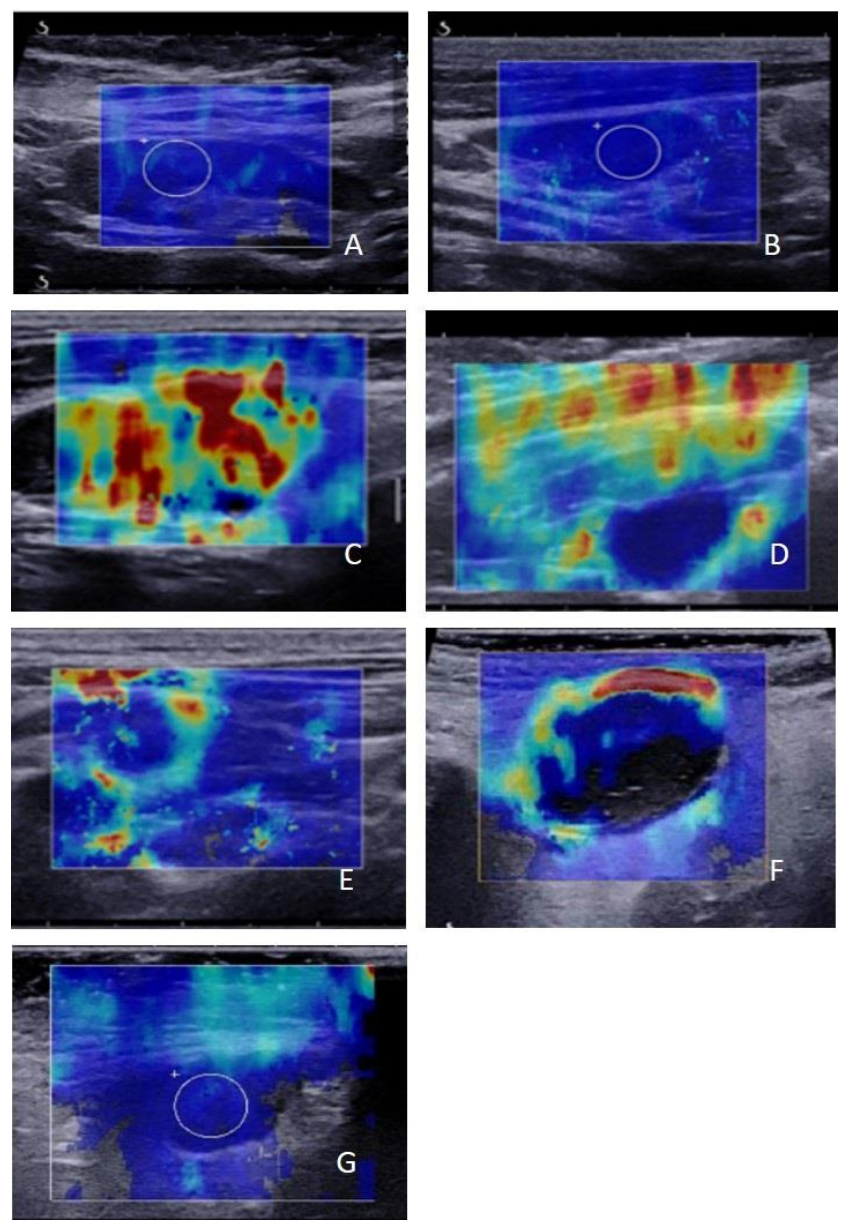

Figure 1

Qualitative SWE patterns: (A-B) Blue pattern. The elasticity map exhibit a globally blue color with Emean values of $11.5 \mathrm{kPa}$ for (A) and $19.4 \mathrm{kPa}$ for (B). (C-D) Nodular pattern. LN stiffer than the surrounding tissues $(\mathrm{C})$ (the internal heterogeneous areas are stiff (yellow and red) whereas the surrounding tissue is mostly soft (blue)) and LN softer than the surrounding tissue (dark blue LN and yellow, red surrounding tissue (D). (E-F) Rim pattern. The LN and the surrounding tissue exhibit the same SWE color map (predominantly blue) with a discontinuous rim of stiffness surrounding the LN (yellow, red). The surrounding tissue is blue whereas the center part of the lymph node is partially void of SWE signal with a surrounding discontinuous stiff rim. (G) Undetermined pattern. Stiff areas in the top part and lack of signal in the lower part of the SWE map with blue LN. Histology: (A) benign LN, (B) B cell lymphoma, (C) metastasis from oropharyngeal carcinoma, (D) benign reactive lymph node, (E) metastasis from lung cancer, $(\mathrm{F}) \mathrm{B}$ cell lymphoma, $(\mathrm{G}) \mathrm{B}$ cell lymphoma. 


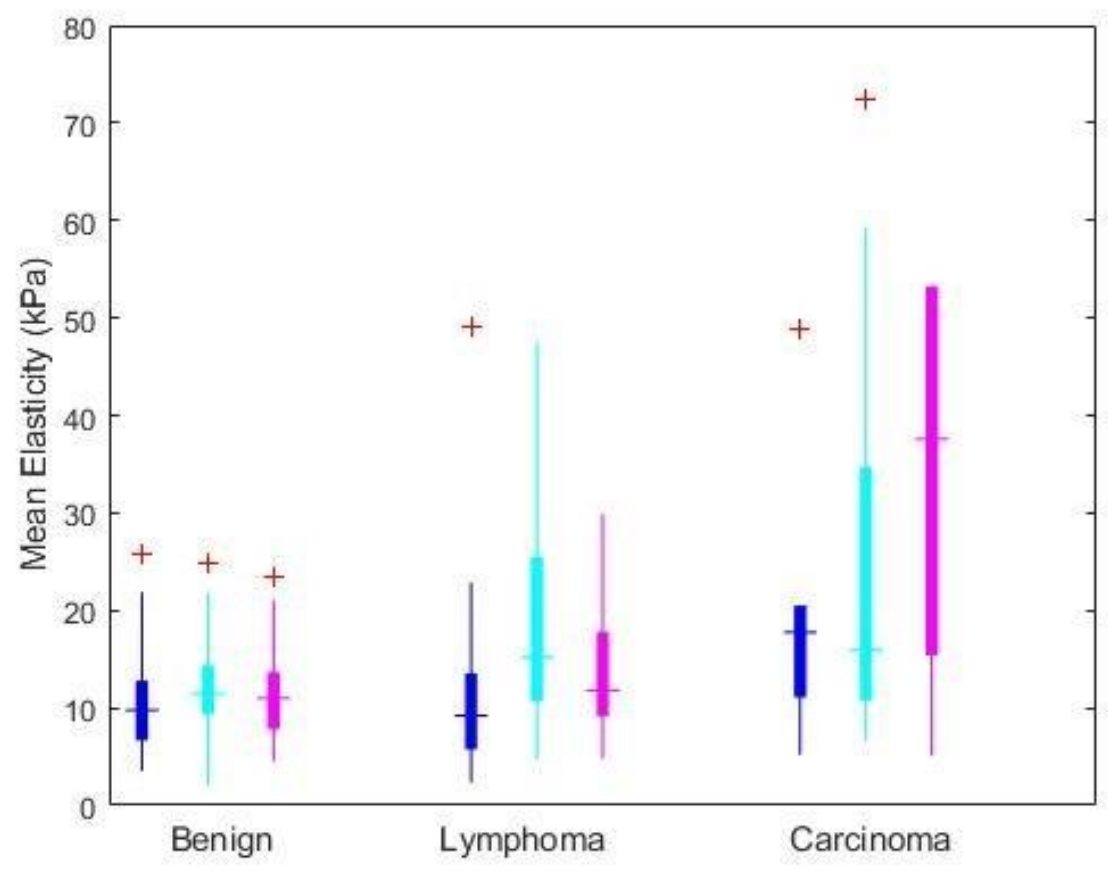

Figure 2: Quantitative SWE estimates

Boxplot diagram of mean elasticity as function of LN histology and localization (blue: Axilla, cyan: Head and Neck, magenta: Inguinal).

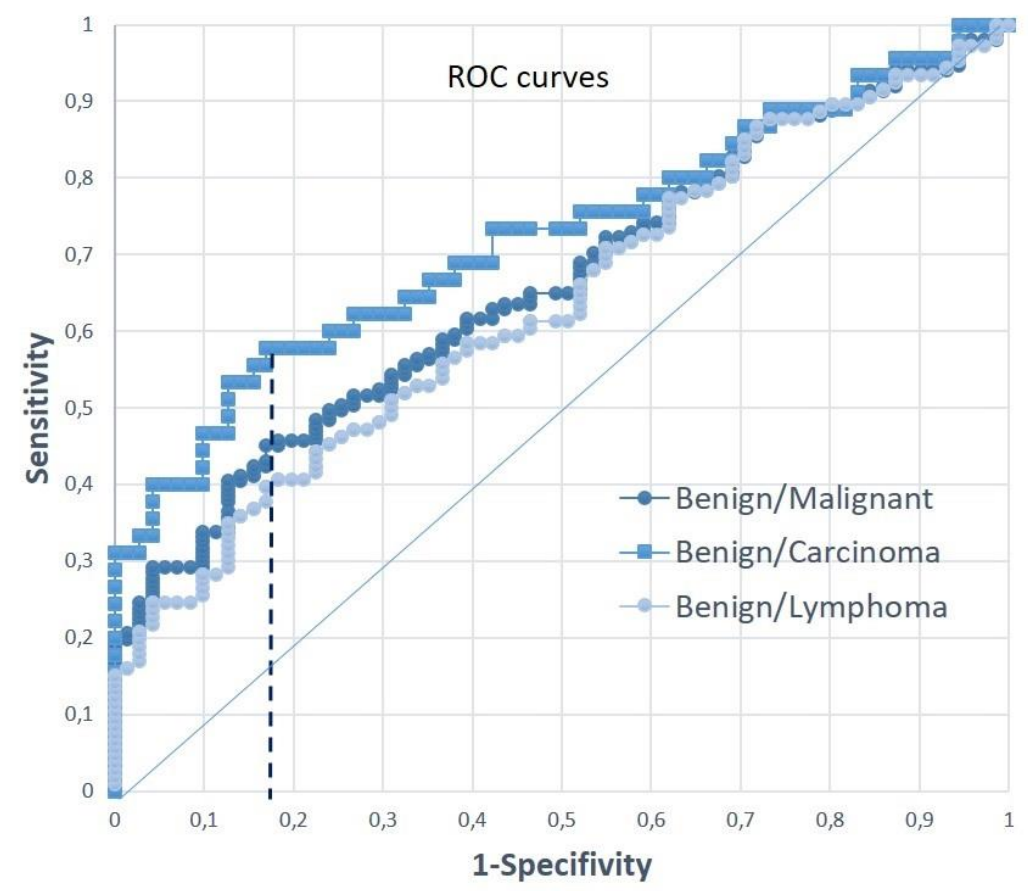

Figure 3: Diagnostic performance of quantitative SWE

Receiver Operating Curves of the quantitative SWE analysis for distinguishing benign from malignant LNs (dark blue), benign LNs from lymphomas (light blue) and benign LNs from carcinomas (medium blue). 


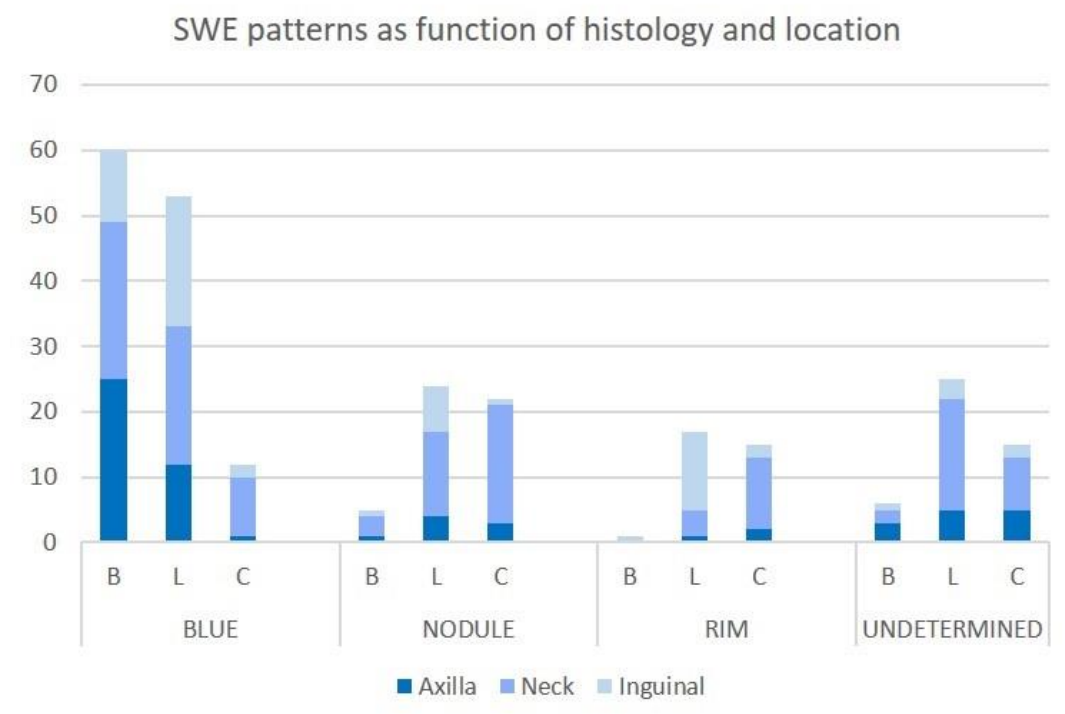

Figure 4: Diagnostic performance of qualitative SWE

Distribution of SWE patterns as function of histology (B: Benign, L: Lymphomas, C: Carcinomas) and location. Nodule, Rim and undetermined patterns were related to malignancy (lymphomas and carcinomas). The blue pattern has a limited interest to distinguish benign LNs and lymphomas. 


\section{Tables}

Table 1: Summary of diagnostic strategies

\begin{tabular}{|c|c|c|}
\hline Strategies & Malignant & Benign \\
\hline Conv-US & $\geq 2$ US Sc & $<2 \mathrm{US} \mathrm{Sc}$ \\
\hline Quant SWE & Emean $\geq \mathrm{E}_{\mathrm{th}}$ & Emean $<\mathrm{E}_{\mathrm{th}}$ \\
\hline Qual SWE & $\operatorname{rim} \underline{\text { or }}$ nod $\underline{\text { or }}$ undt & blue \\
\hline US + Qual SWE & $\geq 2$ US Sc $\underline{\overline{\boldsymbol{o r}}} \operatorname{rim} \underline{\boldsymbol{o r}}$ nod $\underline{\boldsymbol{o r}}$ undt & $<2$ US Sc and blue \\
\hline US + Quant SWE & $\geq 2$ US $\overline{\text { Sc }}$ or Emean $\geq \bar{E}_{\mathrm{th}}$ & $<2$ US Sc and $\overline{\text { Emean }}<\mathrm{E}_{\mathrm{th}}$ \\
\hline $\begin{array}{l}\text { US + Qual SWE + } \\
\text { Quant SWE }\end{array}$ & $\geq 2$ US Sc or rim $\frac{\boldsymbol{o r} \text { nod } \boldsymbol{o r} \text { undt } \underline{\text { or }} \text { Emean }}{\geq \mathrm{E}_{\mathrm{th}}}$ & $<2$ US Sc and blue and Emean $<$ \\
\hline
\end{tabular}

Table 2: Summary of data population and LN localization as function of histology

\begin{tabular}{|c|c|c|c|c|c|}
\hline & & $\begin{array}{l}\text { Benign } \\
(n=71)\end{array}$ & $\begin{array}{l}\text { Lymphomas } \\
\quad(n=106)\end{array}$ & $\begin{array}{l}\text { Carcinomas } \\
\qquad(n=45)\end{array}$ & p-value \\
\hline \multirow[t]{2}{*}{ Population } & Age in year (SD) & $50.1(19.5)$ & $63.3(18.7)$ & $64.2(17.3)$ & $\mathrm{p}<10^{-3}$ \\
\hline & Sex: F/M $(\%$ of F $)$ & $37 / 34(52)$ & $45 / 61(42)$ & $27 / 18(60)$ & ns \\
\hline \multirow[t]{4}{*}{ Localization } & Axilla & 29 & 21 & 9 & \multirow{4}{*}{$\mathrm{p}<0.005$} \\
\hline & Head and neck & 29 & 51 & 27 & \\
\hline & Inguinal & 13 & 30 & 6 & \\
\hline & Other & 0 & 4 & 3 & \\
\hline
\end{tabular}

SD: standard deviation; $\mathrm{F}$ : female ; $\mathrm{M}$ : male ; ns: non-significant:

Table 3: Summary of US and SWE findings as function of histology

\begin{tabular}{|c|c|c|c|c|c|}
\hline & & $\begin{array}{l}\text { Benign } \\
(n=71)\end{array}$ & $\begin{array}{l}\text { Lymphomas } \\
\quad(n=106)\end{array}$ & $\begin{array}{l}\text { Carcinomas } \\
\quad(n=45)\end{array}$ & p-value \\
\hline \multirow[t]{2}{*}{$\begin{array}{c}\text { Conventional } \\
\text { US }\end{array}$} & $\begin{array}{l}\text { Presence of at least two } \\
\text { malignant criteria }\end{array}$ & $\begin{array}{c}19 \\
(26.8 \%)\end{array}$ & $\begin{array}{c}93 \\
(87.7 \%)\end{array}$ & $\begin{array}{c}40 \\
(88.9 \%)\end{array}$ & $\mathrm{p}<10^{-3}$ \\
\hline & $\begin{array}{l}\text { Long / Short axis ratio } \\
\text { (SD) }\end{array}$ & $2.3(0.8)$ & $1.9(0.8)$ & $1.7(0.6)$ & $\mathrm{p}<10^{-3}$ \\
\hline \multirow[t]{3}{*}{$\begin{array}{l}\text { Quantitative } \\
\text { SWE }\end{array}$} & E mean $(\mathrm{SD})$ & $11.4(5.2)$ & $16.7(12.3)$ & $29.5(32.3)$ & $\mathrm{p}<10^{-3}$ \\
\hline & $\mathrm{E} \max (\mathrm{SD})$ & $19.9(9.2)$ & $36.1(33.7)$ & $62(58.2)$ & $\mathrm{p}<10^{-3}$ \\
\hline & $\mathrm{SD}(\mathrm{SD})$ & $3.3(2)$ & $6.4(5.7)$ & $11.1(10.6)$ & $\mathrm{p}<10^{-3}$ \\
\hline \multirow[t]{4}{*}{$\begin{array}{l}\text { Qualitative } \\
\text { SWE }\end{array}$} & $\begin{array}{l}\text { Homogeneous blue } \\
\text { pattern }\end{array}$ & $\begin{array}{c}60 \\
(84.5 \%)\end{array}$ & $\begin{array}{c}55 \\
(51.9 \%)\end{array}$ & $\begin{array}{c}10 \\
(22.2 \%)\end{array}$ & \multirow{4}{*}{$\mathrm{p}<10^{-3}$} \\
\hline & Nodular pattern & $\begin{array}{c}4 \\
(5.6 \%)\end{array}$ & $\begin{array}{c}14 \\
(13.2 \%)\end{array}$ & $\begin{array}{c}9 \\
(20 \%)\end{array}$ & \\
\hline & Rim pattern & $\begin{array}{c}1 \\
(1.4 \%)\end{array}$ & $\begin{array}{c}10 \\
(9.4 \%)\end{array}$ & $\begin{array}{c}13 \\
(28.9 \%)\end{array}$ & \\
\hline & Undetermined pattern & $\begin{array}{c}6 \\
(8.5 \%)\end{array}$ & $\begin{array}{c}27 \\
(25.5 \%) \\
\end{array}$ & $\begin{array}{c}13 \\
(28.9 \%) \\
\end{array}$ & \\
\hline
\end{tabular}

SWE: Shear Wave elastography; SD: standard deviation; 
Table 4: Accuracy (Acc), sensitivity (Se) and specificity (Sp) of conventional US, quantitative and qualitative SWE Differentiation Accuracy Sensitivity Specificity

(\%) (\%) (\%)

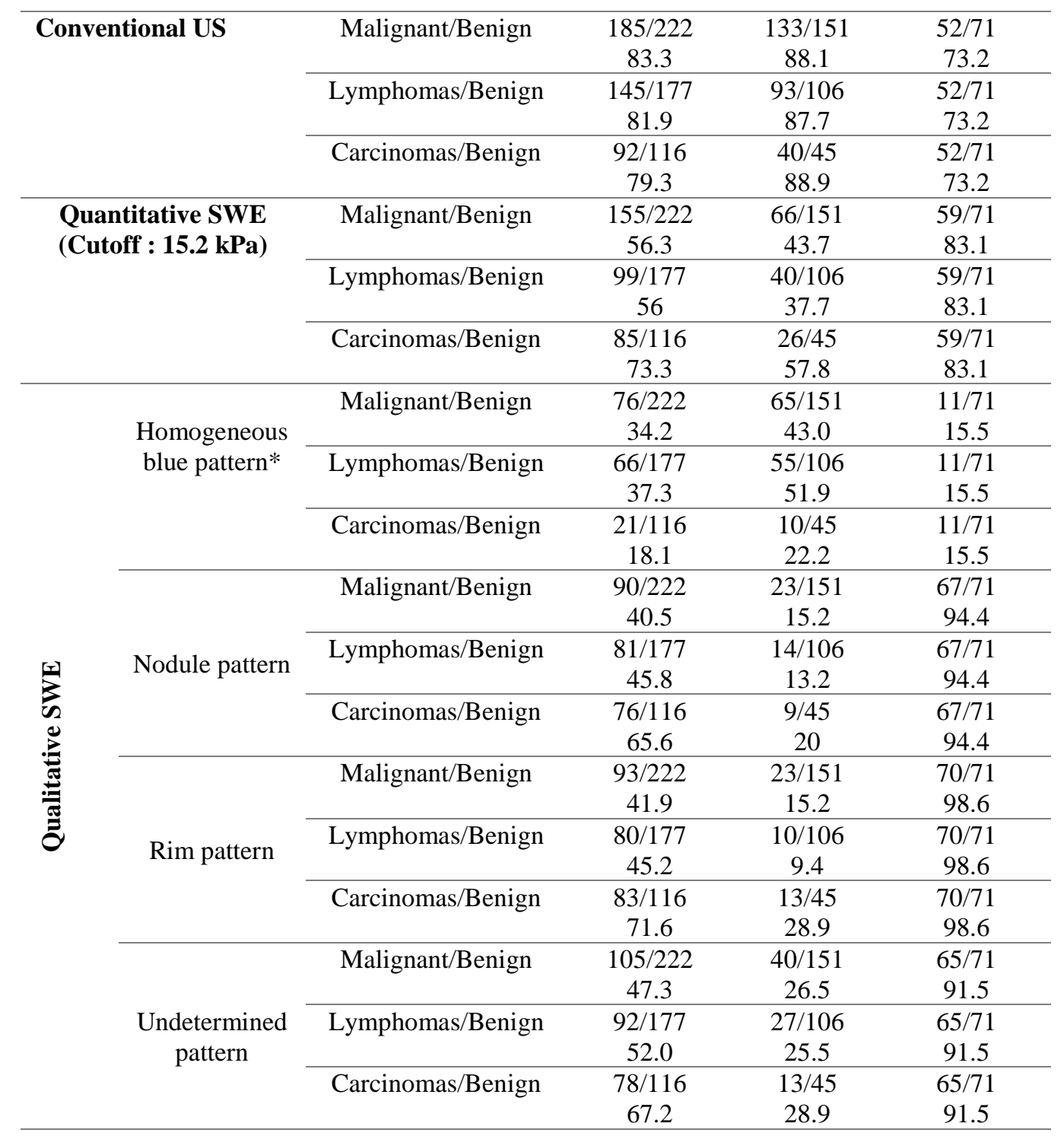

* Accuracy, Sensitivity and Specificity of qualitative SWE were computed considering all patterns as signs of malignancy. Since blue pattern is an indicator of benignity, the complement to $100 \%$ must be considered to access the performance of this index to detect malignancy. 
Table 5 : Mean and (SD) stiffness values in kiloPascals as function of histology and localization

\begin{tabular}{ccccc}
\hline & $\begin{array}{c}\text { Axilla } \\
(\mathbf{n}=59)\end{array}$ & $\begin{array}{c}\text { Head and neck } \\
(\mathbf{n = 1 0 7})\end{array}$ & $\begin{array}{c}\text { Inguinal } \\
(\mathbf{n = 4 9 )}\end{array}$ & p-value \\
\hline $\begin{array}{c}\text { Benign LN } \\
(\mathbf{n = 7 1 )}\end{array}$ & $10.6(5.2)$ & $12.2(5.2)$ & $11.5(5.5)$ & $\mathrm{ns}$ \\
\hline $\begin{array}{c}\text { Lymphomas } \\
(\mathbf{n = 1 0 6 )}\end{array}$ & $11.5(10.1)$ & $20.2(15.8)$ & $14(6.5)$ & $\mathrm{p}<0.005$ \\
\hline $\begin{array}{c}\text { Carcinomas } \\
(\mathbf{n = 4 5})\end{array}$ & $18.5(12.6)$ & $29.1(28.8)$ & $52.6(58.9)$ & $\mathrm{ns}$ \\
\hline $\mathbf{p}$-value & $\mathrm{ns}$ & $\mathrm{p}<0.01$ & $\mathrm{p}<0.04$ & \\
\hline
\end{tabular}

ns : non-significant

Table 6: Diagnostic performances of different diagnostic work-up strategies

\begin{tabular}{lcccccc}
\hline & Conv-US & Qual SWE & $\begin{array}{c}\text { Quant } \\
\text { SWE }\end{array}$ & $\begin{array}{c}\text { Conv-US + } \\
\text { Qual SWE }\end{array}$ & $\begin{array}{c}\text { Conv-US + } \\
\text { Quant SWE }\end{array}$ & $\begin{array}{c}\text { Conv-US+ } \\
\text { Qual / Quant } \\
\text { SWE }\end{array}$ \\
\hline Sensitivity (\%) & 88.1 & 57.0 & 43.71 & 92.0 & 91.4 & 92.7 \\
\hline Specificity (\%) & 73.2 & 84.5 & 83.1 & 67.6 & 67.6 & 63.4 \\
\hline Accuracy (\%) & 83.3 & 67.8 & 56.3 & 84.2 & 83.8 & 83.3 \\
\hline
\end{tabular}

Conv-US : Conventional ultrasound; Quant SWE: Quantitative SWE; Qual SWE: Qualitative SWE 